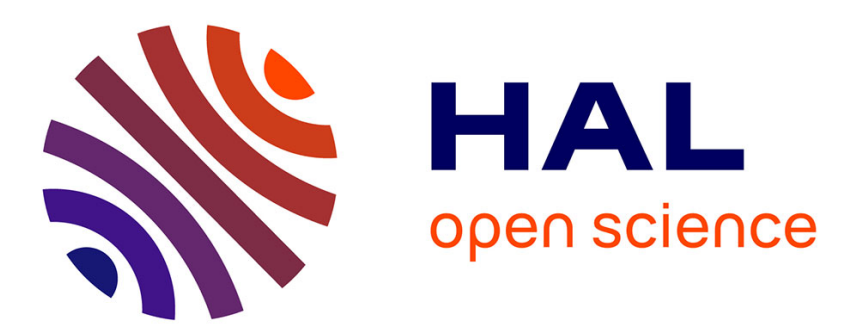

\title{
Stabilization of Linear Hyperbolic Systems of Balance Laws with Measurement Errors
}

\author{
Aneel Tanwani, Christophe Prieur, Sophie Tarbouriech
}

\section{To cite this version:}

Aneel Tanwani, Christophe Prieur, Sophie Tarbouriech. Stabilization of Linear Hyperbolic Systems of Balance Laws with Measurement Errors. S.Tarbouriech, A. Girard, and L. Hetel Control subject to computational and communication constraints, Springer, pp.357-374, 2018, 978-3-319-78449-6. hal01827106

\section{HAL Id: hal-01827106 https://hal.science/hal-01827106}

Submitted on 1 Jul 2018

HAL is a multi-disciplinary open access archive for the deposit and dissemination of scientific research documents, whether they are published or not. The documents may come from teaching and research institutions in France or abroad, or from public or private research centers.
L'archive ouverte pluridisciplinaire HAL, est destinée au dépôt et à la diffusion de documents scientifiques de niveau recherche, publiés ou non, émanant des établissements d'enseignement et de recherche français ou étrangers, des laboratoires publics ou privés. 


\title{
Stabilization of Linear Hyperbolic Systems of Balance Laws with Measurement Errors
}

\author{
Aneel Tanwani, Christophe Prieur, and Sophie Tarbouriech
}

\begin{abstract}
This chapter considers the feedback stabilization of partial differential equations described by linear balance laws when the measurements are subjected to disturbances. Compared to our previous work on robust stabilization of linear hyperbolic systems, the presence of source terms in the system description complicates the analysis. We first consider the case of static controllers, and provide conditions on system data and feedback gain which result in stability of the closed-loop system, and robustness with respect to measurement errors. Motivated by the applications where it is of interest to bound the maximum norm of the state trajectory, we also study feedback stabilization with dynamic controllers. Conditions in terms of matrix inequalities are proposed which lead to robust stability of the closed-loop system in the presence of measurement errors in the feedback. As an application, we study the problem of quantized control, where the quantization error plays the role of disturbance in the measurements. The simulations for an academic example are reported as an illustration of our theoretical results.
\end{abstract}

\section{Introduction}

Balance laws are used to describe the physical systems with certain conservative properties, and hyperbolic partial differential equations (PDEs) provide the mathematical framework to model systems governed by such laws. Stability and stabilization of this class of systems is indeed relevant from several applications viewpoint,

A. Tanwani

LAAS - CNRS, University of Toulouse, CNRS, France. e-mail: tanwani@laas.fr

C. Prieur

GIPSA-lab., CNRS, University of Grenoble, 38000 Grenoble, Francee-mail: christophe.prieur@gipsa-lab.fr

S. Tarbouriech

LAAS - CNRS, University of Toulouse, CNRS, France. e-mail: tarbour@laas.fr 
and several tools are now available for analyzing such properties of hyperbolic systems. We encourage the reader to consult [1] for physical examples of hyperbolic PDEs, and an overview of tools used for studying solutions and stability of this system class.

This chapter concerns the problem of feedback stabilization for a class of boundary controlled hyperbolic systems, which model linear balance laws. We are particularly focused on studying a notion of robust stability when the measurements used for feedback control are subjected to unknown disturbances. In the literature on ordinary differential equations (ODEs), the property of input-to-state stability (ISS), coined in [15], captures the desired robust behavior that we want to study here, while regarding the disturbances as exogenous inputs in the closed-loop system. The Lyapunov function based techniques available for verifying ISS are thus generalized in the context of hyperbolic PDEs in this chapter. Our previous work on robust stabilization of hyperbolic PDEs [17, 18] only considers systems with conservation laws and no source term in the dynamics. Whereas, in this chapter we generalize our results to linear balance laws by including a source term in the PDE. This results in novel stability conditions and calculations.

One finds the Lyapunov stability criteria with $\mathscr{L}^{2}$-norm and dissipative boundary conditions in [2]. Lyapunov stability in $\mathscr{H}^{2}$-norm for nonlinear systems is treated in [3]. Thus, the construction of Lyapunov functions in $\mathscr{H}^{2}$-norm for the hyperbolic PDEs with static control laws can be found in the literature. In the literature, one finds various instances where the ISS related tools are used for stability analysis of interconnected systems. For infinite dimensional systems, the problem of ISS has attracted attention recently but most of the existing works treat the problem with respect to uncertainties in the dynamics. See, for example [11], where a class of linear and bilinear systems is studied. See also [4] where a linearization principle is applied for a class of infinite-dimensional systems in a Banach space. When focusing on parabolic partial differential equations, some works to compute ISS Lyapunov functions have also appeared, such as $[9,10]$. For time-varying hyperbolic PDEs, construction of ISS Lyapunov functions has also been addressed in [13]. The recent work reported in [6,7] derives ISS bounds for 1-D parabolic systems in the presence of boundary disturbances but without the use of Lyapunov-based techniques.

For systems of conservation laws, when seeking robust stabilization with measurement errors, one could see that the results in [5] provide robust stability of $X(\cdot, t)$ in $\mathscr{L}^{2}\left((0,1) ; \mathbb{R}^{n}\right)$ space by using static controllers. The use of dynamic controller for stabilization of systems of conservation laws with ISS estimates in $\mathscr{H}^{1}$-norm and maximum norm is studied in our previous works [17] and [18], respectively. Inspired by the applications of such notions in finite-dimensional systems $[19,16]$, these works also discuss the applications of ISS notion in the context of sampleddata and quantized control of hyperbolic systems, which require stability in a functional space equipped with maximum norm. An intermediate exposition of such applications in finite and infinite dimensions appears in [14].

In this chapter, we build on our works [17] and [18] dealing with input-to-state stabilization in maximum norm and using dynamic feedbacks. The novelty here appears due to the presence of source terms as we migrate from conservation laws 
to balance laws. The presence of source terms induces some changes in the criterion for achieving ISS. The system class and the stability notions of our interest are discussed in Section 2. To clearly highlight the role of the source term, we first deal with the static feedback case and ISS estimates in $\mathscr{L}^{2}$-norm in Section 3. We then develop ISS estimates in supremum norm in Section 4 and carry out the design of a dynamic feedback to achieve that purpose. The applications of these notions in the context of quantized control are discussed in Section 5. Section 6 illustrates the main results of the chapter through an academic example. Finally, some concluding remarks end the chapter in Section 7.

\section{System Class and Stability Notions}

The problem of interest for us is to address feedback control for the class of linear hyperbolic balance laws described by the equation

$$
\frac{\partial X}{\partial t}(z, t)+\Lambda \frac{\partial X}{\partial z}(z, t)=S X(z, t)
$$

where $z \in[0,1]$, and $t \in[0, \infty)$. The matrix $\Lambda$ is assumed to be diagonal and positive definite. The expression $S X(z, t)$ in (1a) denotes the source terms. We call $X:[0,1] \times$ $\mathbb{R}_{+} \rightarrow \mathbb{R}^{n}$ the state trajectory, and the initial condition is defined as

$$
X(z, 0)=X^{0}(z), \quad z \in(0,1)
$$

for some function $X^{0}:(0,1) \rightarrow \mathbb{R}^{n}$. The value of the state $X$ is controlled at the boundary $z=0$ through some input $u: \mathbb{R}_{+} \rightarrow \mathbb{R}^{m}$ so that

$$
X(0, t)=H X(1, t)+B u(t)
$$

where $H \in \mathbb{R}^{n \times n}$ and $B \in \mathbb{R}^{n \times m}$ are constant matrices. We consider the case when only the measurement of the state $X$ at the boundary point $z=1$ is available for each $t \geq 0$. We thus denote the output of the system by

$$
y(t)=X(1, t)+d(t)
$$

where $d \in \mathscr{L}^{\infty}\left([0, \infty), \mathbb{R}^{n}\right)$ is seen as the perturbation in the measurement of the state trajectory at the boundary point.

We are interested in designing a control law $u$ as a function of the output measurement $y$, which stabilizes the system in some appropriate sense. In case there are no perturbations, that is, $d \equiv 0$, one typically chooses $u(t)=K y(t)$. Following this recipe with uncertain measurements, we obtain the closed-loop boundary condition

$$
X(0, t)=(H+B K) X(1, t)+B K d(t)
$$


where $K$ is chosen such that $(H+B K)$ satisfies a certain dissipative condition. We are interested in studying stability of system (1)-(4) with respect to the measurement disturbance $d$.

Definition 1. System (1)-(4) is said to be input-to-state stable in $\mathscr{L}^{2}$-norm $\left(\mathscr{L}^{2}\right.$ ISS) with respect to the disturbance $d$ if there exist constants $c, a>0$ and a class $\mathscr{K}$ function $\gamma$ such that

$$
\|X(z, t)\|_{\mathscr{L}^{2}\left((0,1) ; \mathbb{R}^{n}\right)} \leq c \mathrm{e}^{-a t}\left\|X^{0}\right\|_{\mathscr{L}^{2}\left((0,1) ; \mathbb{R}^{n}\right)}+\gamma\left(\left\|d_{[0, t]}\right\|_{\infty}\right) .
$$

In Section 3, we treat this case and propose conditions for choosing feedback gain $K$, which results in aforementioned stability estimate for the closed-loop system with static control.

Stabilization in $\mathscr{L}^{2}$-norm does not necessarily guarantee convergence of the maximum norm of $X(\cdot, t)$ over the spatial domain $[0,1]$. To do that, we have to consider stability of $X(\cdot, t)$ in $\mathscr{H}^{1}$-norm, which is defined as:

$$
\|X\|_{\mathscr{H}^{1}\left((0,1) ; \mathbb{R}^{n}\right)}:=\left(\|X\|_{\mathscr{L}^{2}\left((0,1) ; \mathbb{R}^{n}\right)}^{2}+\|\partial X\|_{\mathscr{L}^{2}\left((0,1) ; \mathbb{R}^{n}\right)}^{2}\right)^{1 / 2} .
$$

The following proposition, proved in [18], allows us to make the connection between $\mathscr{C}^{0}$-norm and $\mathscr{H}^{1}$-norm.

Proposition 1. Given any function $X:[0,1] \rightarrow \mathbb{R}^{n}$ such that $X \in \mathscr{C}^{0}\left([0,1] ; \mathbb{R}^{n}\right) \cap$ $\mathscr{H}^{1}\left((0,1) ; \mathbb{R}^{n}\right)$. It holds that, for every $z \in[0,1]$,

$$
\max _{z \in[0,1]}|X(z)|^{2} \leq|X(0)|^{2}+\|X\|_{\mathscr{H}^{1}\left((0,1) ; \mathbb{R}^{n}\right)}^{2}
$$

By definition, functions with finite $\mathscr{H}^{1}$-norm must be differentiable Lebesgue almost everywhere, and since $d \in \mathscr{L}^{\infty}$, we can no longer use static feedbacks. The use of dynamic controller allows us to circumvent this problem; see [18] for details. The dynamic controller driven by the output $y$ that we choose for our purposes is described by the following equations:

$$
\begin{aligned}
\dot{\eta}(t) & =-\alpha(\eta(t)-y(t))=-\alpha \eta(t)+\alpha X(1, t)+\alpha d(t) \\
\eta(0) & =\eta^{0} \\
u(t) & =K \eta(t),
\end{aligned}
$$

where $\eta^{0} \in \mathbb{R}^{n}$ is the initial condition for the controller dynamics.

Definition 2. System (1), (2), (7) is said to be input-to-state stable in $\mathscr{C}^{0}$-norm $\left(\mathscr{C}^{0}\right.$ ISS) with respect to the disturbance $d$ if there exist constants $c, a>0$ and a class $\mathscr{K}$ function $\gamma$ such that

$$
\max _{z \in[0,1]}|X(z, t)| \leq c \mathrm{e}^{-a t} M_{X^{0}, \eta^{0}}+\gamma\left(\left\|d_{[0, t]}\right\|_{\infty}\right) .
$$


In (8), $M_{X^{0}, \eta^{0}}$ is a constant that depends on some norm associated with the function $X^{0}$ and the initial state $\eta^{0}$ chosen for the dynamic compensator $\eta$. We are interested in designing $\alpha, K$ such that the closed-loop system (1), (2), (7) is stable in the sense of Definition 2.

In terms of analysis, the addition of dynamic controller introduces a coupling of ODEs and PDEs in the closed loop, which makes the analysis more challenging. The derivations of the main results are also more involved compared to [18] due to the presence of source terms. We use Lyapunov function based analysis to synthesize the controller and guarantee ISS with respect to the perturbation $d$. After designing controllers which achieve the desired ISS estimates, we study an application of these notions in the context of quantized control: We establish practical stability of the system, and derive ultimate bounds on the state trajectory in terms of the quantization error. The problem of quantized control has mostly been studied in finite-dimensional systems so far $[8,12,14,17]$, and this chapter extends this problem setting to the case of hyperbolic balance laws.

\section{Static Control and $\mathscr{L}^{2}$-estimates}

We first address the problem of finding conditions for the system to be $\mathscr{L}^{2}$-ISS as formulated in Definition 1. In the following, $\mathscr{D}_{+}^{n}$ denotes the set of diagonal positive definite matrices

Theorem 1. If there exist scalars $\kappa \in(0,1), c<\lambda_{\min }(\Lambda)$, a matrix $D \in \mathscr{D}_{+}^{n}$, and $a$ matrix $K \in \mathbb{R}^{m \times n}$, such that

$$
\begin{gathered}
(H+B K)^{\top} \Lambda D(H+B K) \leq \kappa \Lambda D \\
S^{\top} D+D S \leq c \log \left(\frac{1}{\kappa}\right) D,
\end{gathered}
$$

then system (1)-(2) with $u=K y$ is ISS with respect to the disturbance $d$.

Proof. The proof is based on introducing a Lyapunov function and analyze its derivative with respect to time. As a candidate, we choose $V: \mathscr{L}^{2}\left((0,1) ; \mathbb{R}^{n}\right) \rightarrow \mathbb{R}_{+}$ given by

$$
V(X):=\int_{0}^{1} X^{\top}(z) D X(z) \mathrm{e}^{-\mu z} d z,
$$

where $D$ is a diagonal positive definite matrix satisfying (9). The constant $\mu>0$ is chosen such that

$$
\frac{c}{\lambda_{\min }(\Lambda)} \log \left(\frac{1}{\kappa}\right)<\mu<\log \left(\frac{1}{\kappa}\right),
$$

which is possible because $c<\lambda_{\min }(\Lambda)$.

Using an integration by parts, along the solutions to (1)-(2) with $u=K y$, the time derivative of $V$ yields 


$$
\begin{aligned}
\dot{V} & =\int_{0}^{1}\left(\partial_{t} X^{\top} D X+X^{\top} D \partial_{t} X\right) e^{-\mu z} d z \\
& =-\int_{0}^{1}\left(\partial_{z} X^{\top} \Lambda D X+X^{\top} D \Lambda \partial_{z} X\right) e^{-\mu z} d z+\int_{0}^{1} X^{\top}\left(S^{\top} D+D S\right) X e^{-\mu z} d z \\
& \leq-\left[X^{\top} \Lambda D X e^{-\mu z}\right]_{0}^{1}-\mu \int_{0}^{1} X^{\top} D \Lambda X e^{-\mu z} d z+c \log \left(\frac{1}{\kappa}\right) \int_{0}^{1} X^{\top} D X e^{-\mu z} d z \\
& \leq-e^{-\mu} X(1, t)^{\top} \Lambda D X(1, t)+X(0, t)^{\top} \Lambda D X(0, t)-\sigma V,
\end{aligned}
$$

where $\sigma:=\left(\mu \lambda_{\min }(\Lambda)-c \log (1 / \kappa)\right)>0$ due to the first inequality in (10). We now substitute the expression for boundary control to get

$$
X(0, t)=(H+B K) X(1, t)+B K d(t)
$$

Using (9a), we get

$$
\begin{aligned}
\dot{V}(t) & \leq-\sigma V(t)-\left(\mathrm{e}^{-\mu}-\kappa\right) X^{\top}(1, t) \Lambda D X(1, t)+d(t)^{\top} \Lambda D d(t) \\
& \leq-\sigma V(t)+\chi d(t)^{\top} d(t)
\end{aligned}
$$

where $\chi=\lambda_{\max }(\Lambda D)$, and $\mathrm{e}^{-\mu}>\kappa$ due to the second inequality in (10). The ISS estimate now follows from the last inequality.

\section{Dynamic Control and $\mathscr{C}^{0}$-estimates}

In this section, we are interested in analyzing the closed-loop system (1), (2), (7). Since we are interested in computing estimates on the $\mathscr{H}^{1}$-norm of the state $X$, we need to look at the evolution of $X_{z}$. We recall that, for the closed-loop system with dynamic controller, the state trajectory $X$ satisfies

$$
\begin{gathered}
X_{t}(z, t)+\Lambda X_{z}(z, t)=S X(z, t), \\
X(z, 0)=X^{0}(z), \forall z \in[0,1], \\
X(0, t)=H X(1, t)+B K \eta(t) .
\end{gathered}
$$

For what follows, we are also interested in analyzing the dynamics of $X_{z}:=\partial_{z} X$ which are derived as follows:

$$
\frac{\partial X_{z}}{\partial t}(z, t)+\Lambda \frac{\partial X_{z}}{\partial z}(z, t)=S X_{z}(z, t)
$$

To obtain the boundary condition for $X_{z}$, from (11c), we have

$$
X_{t}(0, t)=H X_{t}(1, t)+B K \dot{\eta}(t) .
$$

Substituting $X_{t}(z, t)=-\Lambda X_{z}(z, t)+S X(z, t)$ for each $z \in[0,1]$, we get 


$$
\begin{aligned}
\Lambda X_{z}(0, t)= & H \Lambda X_{z}(1, t)+(S H-H S) X(1, t)+S B K \eta(t)-B K \dot{\eta}(t) \\
= & H \Lambda X_{z}(1, t)+S(H+B K) X(1, t)+S B K(\eta(t)-X(1, t))-H S X(1, t) \\
& -B K\left(\dot{\eta}(t)-X_{t}(1, t)\right)+B K\left(\Lambda X_{z}(1, t)-S X(1, t)\right) \\
= & (H+B K) \Lambda X_{z}(1, t)+[S(H+B K)-(H+B K) S] X(1, t) \\
& +S B K(\eta(t)-X(1, t))-B K\left(\dot{\eta}(t)-X_{t}(1, t)\right) \\
= & -(H+B K) X_{t}(1, t)-B K\left(\dot{\eta}(t)-X_{t}(1, t)\right)+S(H+B K) X(1, t) \\
& +S B K(\eta(t)-X(1, t)) .
\end{aligned}
$$

using (11a) for the last equality. We now use these equations as the system description and proceed to develop the next result.

\subsection{Stability Result}

The second main contribution of this chapter is to present conditions on the controller dynamics (7) which results in stability of system (1)-(2), and robustness with respect to the measurement disturbances $d$ in the sense of Definition 2. To state the result, we introduce some notation. For scalars $\mu>0$ and $0<\kappa<1$, let $\rho:=e^{-\mu}-\kappa$; let $F:=B K$, and $Q:=F^{\top} \Lambda D F$ for $D \in \mathscr{D}_{+}^{n}$; and finally, let $G:=H^{\top} \Lambda D F$. We introduce the matrices

$$
\Omega_{1}:=\left[\begin{array}{ccc}
\rho \beta_{1} \Lambda D & -\beta_{1}(G+Q) & 0 \\
* & 2 \alpha \beta_{3} I-\left(\beta_{1}+\alpha^{2} \beta_{2}\right) Q & \beta_{3} I+\alpha \beta_{2} G \\
* & * & \beta_{2}\left(\rho \Lambda D+Q+G+G^{\top}\right)
\end{array}\right]
$$

and with $\widetilde{S}:=S(H+F)$,

$$
\Omega_{2}:=\beta_{2}\left[\begin{array}{ccc}
-\widetilde{S}^{\top} \Lambda D \widetilde{S} & -\widetilde{S}^{\top} \Lambda D S F & H^{\top} \Lambda D \widetilde{S} \\
* & -F^{\top}\left(S^{\top} \Lambda D S+\Lambda D S+S^{\top} \Lambda D\right) F & H^{\top} \Lambda D S F \\
* & * & 0
\end{array}\right]
$$

in which $\alpha, \beta_{1}, \beta_{2}, \beta_{3}$ are some positive constants, and $*$ denotes the transposed matrix block. It must be noted that the matrix $\Omega_{2}=0$ if there is no source term, that is, $S=0$. In the following statement, we denote the induced-Euclidean norm of a matrix $M$ by $\|M\|_{2}$.

Theorem 2. Assume that there exist scalars $\mu \in \mathbb{R}_{+}, \kappa \in(0,1)$, a matrix $D \in \mathscr{D}_{+}^{n}$, the gain matrix $K$, and the positive constants $\alpha, \beta_{1}, \beta_{2}, \beta_{3}$ in the definitions of $\Omega_{1}$ and $\Omega_{2}$ such that 


$$
\begin{gathered}
(H+B K)^{\top} \Lambda D(H+B K) \leq \kappa \Lambda D \\
S^{\top} D+D S<\mu \lambda_{\min }(\Lambda) D, \\
S^{\top} \Lambda^{2} D+\Lambda^{2} D S \leq \mu \lambda_{\min }(\Lambda) \Lambda^{2} D, \\
\Omega_{1}+\Omega_{2}>0
\end{gathered}
$$

Then, the closed-loop system satisfies the ISS estimate (8) with

$$
M_{X^{0}}:=\left\|X^{0}\right\|_{\mathscr{H}^{1}\left((0,1) ; \mathbb{R}^{n}\right)}^{2}+\left|\eta^{0}-X(1,0)\right|^{2} .
$$

Remark 1. Condition (14) provides a generalization of stability conditions that were studied earlier in $[17,18]$ in the sense that (14a) and (14d) (with $\Omega_{2}=0$ ) were already proposed there. Conditions (14b) and (14c) appear because of the non-zero source term $S X$. If $S$ is symmetric, then (14b) and (14c) are equivalent.

The proof of Theorem 2 is based on constructing a Lyapunov function for the closed-loop system (1), (2), (7). Within the remainder of this section, we provide this construction, and the analysis involving the computation of the derivative of this function. The required ISS estimate then follows from the condition (14) and the bound on the derivative of the Lyapunov function constructed.

\subsection{Construction of the Lyapunov Function}

As a candidate, we choose $V: \mathscr{H}^{1}\left((0,1) ; \mathbb{R}^{n}\right) \times \mathbb{R}^{n} \rightarrow \mathbb{R}_{+}$given by

$$
V:=V_{1}+V_{2}+V_{3}
$$

where $V_{1}: \mathscr{H}^{1}\left((0,1) ; \mathbb{R}^{n}\right) \rightarrow \mathbb{R}_{+}$is defined as,

$$
V_{1}(X):=\int_{0}^{1} X(z)^{\top} P_{1} X(z) e^{-\mu z} d z
$$

where we choose $P_{1}:=\beta_{1} D$, and $\beta_{1}, D$ satisfy (14). Similarly, $V_{2}: \mathscr{H}^{1}\left((0,1) ; \mathbb{R}^{n}\right) \rightarrow$ $\mathbb{R}_{+}$is given by

$$
V_{2}(X):=\int_{0}^{1} \partial X(z)^{\top} P_{2} \partial X(z) e^{-\mu z} d z
$$

with $P_{2}=\beta_{2} \Lambda^{2} D$, and finally $V_{3}: \mathscr{H}^{1}\left((0,1) ; \mathbb{R}^{n}\right) \times \mathbb{R}^{n} \rightarrow \mathbb{R}_{+}$is given by

$$
V_{3}(X, \eta)=(\eta-X(1))^{\top} P_{3}(\eta-X(1)) .
$$

with $P_{3}=\beta_{3} I$. With this choice of $V$, we can now introduce the constants $\underline{c}_{P}:=$ $e^{-\mu} \min _{i=1,2,3}\left\{\lambda_{\min }\left(P_{i}\right)\right\}, \bar{c}_{P}:=\max _{i=1,2,3}\left\{\lambda_{\max }\left(P_{i}\right)\right\}$ such that, for all $X \in \mathscr{H}^{1}\left((0,1) ; \mathbb{R}^{n}\right)$, and $\eta \in \mathbb{R}^{n}$, 


$$
\begin{aligned}
\underline{c}_{P}\left(\|X\|_{\mathscr{H}^{1}\left((0,1) ; \mathbb{R}^{n}\right)}^{2}+|\eta-X(1)|^{2}\right) \leq V(X, \eta) \leq \\
\bar{c}_{P}\left(\|X\|_{\mathscr{H}^{1}\left((0,1) ; \mathbb{R}^{n}\right)}^{2}+|\eta-X(1)|^{2}\right) .
\end{aligned}
$$

\subsection{Lyapunov Dissipation Inequality}

We now derive the bound on $\dot{V}$ that was used in Section 4.2 to obtain the desired ISS estimate. This is done by analyzing the time derivative of each of the three functions in the definition of the Lyapunov function.

\section{Analyzing $V_{1}$ :}

Using an integration by parts and recalling that $P_{1}=\beta_{1} D$ is a diagonal positive definite matrix, the time derivative of $V_{1}$ yields

$$
\begin{aligned}
\dot{V}_{1} & =\beta_{1} \int_{0}^{1}\left(\partial_{t} X^{\top} D X+X^{\top} D \partial_{t} X\right) e^{-\mu z} d z \\
& =-\beta_{1} \int_{0}^{1}\left(\partial_{z} X^{\top} \Lambda D X+X^{\top} D \Lambda \partial_{z} X\right) e^{-\mu z} d z+\beta_{1} \int_{0}^{1} X^{\top}\left(S^{\top} D+D S\right) X e^{-\mu z} d z \\
& \leq-\beta_{1}\left[X^{\top} \Lambda D X e^{-\mu z}\right]_{0}^{1}-\beta_{1} \mu \int_{0}^{1} X^{\top} \Lambda D X e^{-\mu z} d z+\beta_{1} v \int_{0}^{1} X^{\top} D X e^{-\mu z} d z \\
& \leq-\beta_{1} e^{-\mu} X(1, t)^{\top} D \Lambda X(1, t)+\beta_{1} X(0, t)^{\top} D \Lambda X(0, t)-\sigma_{1} V_{1}
\end{aligned}
$$

where $v<\lambda_{\min }(\Lambda)$ due to (14b) which results in $\sigma_{1}:=\beta_{1}\left(\mu \lambda_{\min }(\Lambda)-v\right)$ strictly positive. We now impose the boundary conditions by substituting the value of control $u$ given in (11c) to get

$$
X(0, t)=(H+B K) X(1, t)+B K(\eta-X(1, t))
$$

which results in

$$
\begin{aligned}
\dot{V}_{1} \leq & -\sigma_{1} V_{1}-\beta_{1} e^{-\mu} X(1, t)^{\top} \Lambda D X(1, t) \\
& +\beta_{1} X(1, t)^{\top}(H+B K)^{\top} \Lambda D(H+B K) X(1, t) \\
& +2 \beta_{1} X(1, t)^{\top}(H+B K)^{\top} \Lambda D B K(\eta-X(1, t)) \\
& +\beta_{1}(\eta-X(1, t))^{\top} K^{\top} B^{\top} \Lambda D B K(\eta-X(1, t)) .
\end{aligned}
$$

With (14a), we thus get

$$
\begin{aligned}
\dot{V}_{1} \leq-\sigma_{1} V_{1}- & \beta_{1}\left(e^{-\mu}-\kappa\right) X(1, t)^{\top} \Lambda D X(1, t) \\
+2 \beta_{1} X(1, t)^{\top}(H+B K)^{\top} \Lambda D B K(\eta-X(1, t)) & +\beta_{1}(\eta-X(1, t))^{\top} K^{\top} B^{\top} \Lambda D B K(\eta-X(1, t)) .
\end{aligned}
$$


Analyzing $V_{2}$ :

Using (12) and repeating the same calculations as in the case of $\dot{V}_{1}$ when obtaining (18), we get

$$
\dot{V}_{2} \leq-\beta_{2} e^{-\mu} X_{z}(1, t)^{\top} \Lambda^{2} D \Lambda X_{z}(1, t)+\beta_{2} X_{z}(0, t)^{\top} \Lambda^{2} D \Lambda X_{z}(0, t)-\sigma_{2} V_{2},
$$

where $\sigma_{2}:=\beta_{2}\left(\mu \lambda_{\min }(\Lambda)-v\right)>0$ by our choice of $v$. Substitute the value of $\Lambda X_{z}(0, t)$ from (13) results in

$$
\dot{V}_{2} \leq-\sigma_{2} V_{2}-\beta_{2} e^{-\mu} X_{z}(1, t)^{\top} \Lambda^{2} D \Lambda X_{z}(1, t)+\beta_{2}\left(T_{1}+T_{2}+T_{3}\right)
$$

where

$$
\begin{aligned}
T_{1} & :=\left[(H+F) X_{t}(1, t)+F\left(\dot{\eta}-X_{t}(1, t)\right)\right]^{\top} \Lambda D\left[(H+F) X_{t}(1, t)+F\left(\dot{\eta}-X_{t}(1, t)\right)\right] \\
T_{2} & :=[\widetilde{S} X(1, t)+S F(\eta(t)-X(1, t))]^{\top} \Lambda D[\widetilde{S} X(1, t)+S F(\eta(t)-X(1, t))] \\
T_{3} & :=2\left[-(H+F) X_{t}(1, t)-F\left(\dot{\eta}-X_{t}(1, t)\right)\right]^{\top} \Lambda D[\widetilde{S} X(1, t)+S F(\eta(t)-X(1, t))] \\
& =-2\left[(H+F) X_{t}(1, t)+F\left(\dot{\eta}-X_{t}(1, t)\right)\right]^{\top} \Lambda D[\widetilde{S} X(1, t)+S F(\eta(t)-X(1, t))]
\end{aligned}
$$

and we used the notation $\widetilde{S}=S(H+F)$ and $F=B K$. The term $T_{1}$ is already analyzed in a manner similar to our paper [18], whereas the terms $T_{2}$ and $T_{3}$ appear only because of the source term $S$, which is considered in this paper. After substituting $\eta$-dynamics in (7a), straightforward calculations yield

$$
\begin{aligned}
T_{1}= & X_{t}(1, t)^{\top}\left[(H+F)^{\top} \Lambda D(H+F)-H^{\top} \Lambda D F-F^{\top} \Lambda D H-F^{\top} \Lambda D F\right] X_{t}(1, t) \\
& +\alpha^{2}(\eta(t)-X(1, t))^{\top} F^{\top} \Lambda D F(\eta(t)-X(1, t)) \\
& -2 \alpha X_{t}(1, t)^{\top} H^{\top} \Lambda D F(\eta-X(1, t))-2 \alpha^{2}(\eta(t)-X(1, t))^{\top} F^{\top} \Lambda D F d(t) \\
& +2 \alpha X_{t}(1, t)^{\top} H^{\top} \Lambda D F d(t)+\alpha^{2} d^{\top} F^{\top} \Lambda D F d(t) .
\end{aligned}
$$

Similarly, for $T_{2}$, we get

$$
\begin{aligned}
T_{2}= & X(1, t)^{\top} \widetilde{S}^{\top} \Lambda D \widetilde{S} X(1, t)+2 X(1, t)^{\top} \widetilde{S}^{\top} \Lambda D S F(\eta(t)-X(1, t)) \\
& +(\eta(t)-X(1, t))^{\top} F^{\top} S^{\top} \Lambda D S F(\eta(t)-X(1, t)),
\end{aligned}
$$

and finally, $T_{3}$ yields

$$
\begin{aligned}
T_{3}= & 2 \alpha(\eta(t)-X(1, t))^{\top} F^{\top} \Lambda D S F(\eta(t)-X(1, t)) \\
& -2 X_{t}(1, t)^{\top} H^{\top} \Lambda D \widetilde{S} X(1, t)-2 X_{t}(1, t)^{\top} H^{\top} \Lambda D S F(\eta(t)-X(1, t)) \\
& +2 \alpha(\eta(t)-X(1, t))^{\top} F^{\top} \Lambda D \widetilde{S} X(1, t) \\
& -2 \alpha X(1, t)^{\top} \widetilde{S}^{\top} \Lambda D F d(t)+2 \alpha(\eta(t)-X(1, t))^{\top} F^{\top} S^{\top} \Lambda D F d(t) .
\end{aligned}
$$

One can use the Young's inequality to bound the terms with disturbances $d$, so that, for every $\bar{\zeta}>0$, the disturbance terms in $\beta_{2} T_{1}$ are bounded as 
Feedback Stabilization of Linear Hyperbolic Systems

$$
\begin{gathered}
\left|2 \alpha^{2} \beta_{2}(\eta-X(1, t))^{\top} F^{\top} \Lambda D F d(t)\right| \leq \frac{\bar{\zeta}}{4}|\eta-X(1, t)|^{2}+4\left(\alpha^{2} \beta_{2}\right)^{2} \frac{\left\|F^{\top} \Lambda D F\right\|_{2}^{2}}{\bar{\zeta}}|d(t)|^{2} \\
\left|2 \alpha \beta_{2} X_{t}(1, t)^{\top} H^{\top} \Lambda D F d(t)\right| \leq \bar{\zeta}\left|X_{t}(1, t)\right|^{2}+\left(\alpha \beta_{2}\right)^{2} \frac{\left\|H^{\top} \Lambda D F\right\|_{2}^{2}}{\bar{\zeta}}|d(t)|^{2}
\end{gathered}
$$

and the terms in $\beta_{2} T_{3}$ can be bounded as

$$
\begin{aligned}
&\left|2 \alpha \beta_{2} X(1, t)^{\top} \widetilde{S}^{\top} \Lambda D F d(t)\right| \leq \bar{\zeta}|X(1, t)|^{2}+\left(\alpha \beta_{2}\right)^{2} \frac{\left\|\widetilde{S}^{\top} \Lambda D F\right\|_{2}^{2}}{\bar{\zeta}}|d(t)|^{2}, \\
&\left|2 \alpha \beta_{2}(\eta(t)-X(1, t))^{\top} F^{\top} S^{\top} \Lambda D F d(t)\right| \leq \frac{\bar{\zeta}}{4}|\eta-X(1, t)|^{2} \\
&+4\left(\alpha \beta_{2}\right)^{2} \frac{\left\|F^{\top} S^{\top} \Lambda D F\right\|_{2}^{2}}{\bar{\zeta}}|d(t)|^{2} .
\end{aligned}
$$

With (20), using (9a) and (13), we get

$$
\begin{aligned}
\dot{V}_{2} \leq & -\sigma V_{2}-\beta_{2} X_{t}(1, t)^{\top}\left[\left(\mathrm{e}^{-\mu}-\kappa\right) \Lambda D+H^{\top} \Lambda D F+F^{\top} \Lambda D H+F^{\top} \Lambda D F\right] X_{t}(1, t) \\
& +\beta_{2}(\eta(t)-X(1, t))^{\top} F^{\top}\left[\alpha^{2} \Lambda D+S^{\top} \Lambda D S+\Lambda D S+S^{\top} \Lambda D\right] F(\eta(t)-X(1, t)) \\
& +\beta_{2} X(1, t)^{\top} \widetilde{S}^{\top} \Lambda D \widetilde{S} X(1, t)-2 \beta_{2} X_{t}(1, t)^{\top} H^{\top} \Lambda D \widetilde{S} X(1, t) \\
& -2 \beta_{2} X_{t}(1, t)^{\top}\left[\alpha(H+F)^{\top} \Lambda D F+H^{\top} \Lambda D S F\right](\eta-X(1, t)) \\
& +2 \beta_{2} X(1, t)^{\top}\left[\widetilde{S}^{\top} \Lambda D S F+\alpha F^{\top} \Lambda D \widetilde{S}\right](\eta(t)-X(1, t)) \\
& +\bar{\zeta}|X(1, t)|^{2}+\bar{\zeta}\left|X_{t}(1, t)\right|^{2}+\frac{\bar{\zeta}}{2}|\eta(t)-X(1, t)|^{2}+\chi_{1}|d(t)|^{2}
\end{aligned}
$$

where

$$
\begin{aligned}
\chi_{1}:= & \alpha^{2}\left\|F^{\top} \Lambda D F\right\|_{2}^{2}+4 \alpha^{4} \beta_{2}^{2} \frac{\left\|F^{\top} \Lambda D F\right\|_{2}^{2}}{\bar{\zeta}}+\left(\alpha \beta_{2}\right)^{2} \frac{\left\|H^{\top} \Lambda D F\right\|_{2}^{2}}{\bar{\zeta}} \\
& +4\left(\alpha \beta_{2}\right)^{2} \frac{\left\|F^{\top} S^{\top} \Lambda D F\right\|_{2}^{2}}{\bar{\zeta}}+\left(\alpha \beta_{2}\right)^{2} \frac{\left\|\widetilde{S}^{\top} \Lambda D F\right\|_{2}^{2}}{\bar{\zeta}} .
\end{aligned}
$$

Analyzing $V_{3}$ :

Once again, substituting the dynamics of $\eta$ from (7a) in the expression of $\dot{V}_{3}$ to get

$$
\begin{aligned}
\dot{V}_{3}=2 \beta_{3}(\eta(t)-X(1, t))^{\top}\left(\dot{\eta}(t)-X_{t}(1, t)\right) \\
=-2 \alpha \beta_{3}|(\eta(t)-X(1, t))|^{2}-2 \beta_{3}(\eta(t)-X(1, t))^{\top} X_{t}(1, t) \\
\quad+2 \alpha \beta_{3}(\eta(t)-X(1, t))^{\top} d(t) .
\end{aligned}
$$


Once again, Young's inequality is used to obtain, $\forall \bar{\zeta}>0$

$$
2 \alpha \beta_{3}(\eta(t)-X(1, t))^{\top} d(t) \leq \frac{\bar{\zeta}}{4}|\eta(t)-X(1, t)|^{2}+\frac{4\left(\alpha \beta_{3}\right)^{2}}{\bar{\zeta}}|d(t)|^{2},
$$

which further yields

$$
\begin{aligned}
\dot{V}_{3} \leq & -\left(2 \alpha \beta_{3}+\frac{\bar{\zeta}}{4}\right)|(\eta(t)-X(1, t))|^{2}-2 \beta_{3}(\eta(t)-X(1, t))^{\top} X_{t}(1, t) \\
& +\frac{\bar{\zeta}}{2}|(\eta(t)-X(1, t))|^{2}+\frac{4\left(\alpha \beta_{3}\right)^{2}}{\bar{\zeta}}|d(t)|^{2} .
\end{aligned}
$$

Combining $\dot{V}_{1}, \dot{V}_{2}, \dot{V}_{3}$ :

By introducing the vector $w$ as

$$
w(t):=\left(X(1, t)^{\top},(\eta(t)-X(1, t))^{\top}, X_{t}^{\top}(1, t)\right)^{\top},
$$

one can manage the terms in the expressions for $\dot{V}_{i}, i=1,2,3$ to get

$$
\dot{V} \leq-\sigma_{1} V_{1}-\sigma_{2} V_{2}-\frac{\bar{\zeta}}{4} V_{3}-w^{\top}\left(\Omega_{1}+\Omega_{2}\right) w+\bar{\zeta} w^{\top} w+\chi|d(t)|^{2}
$$

where the constant $\chi$ is given by

$$
\chi:=\chi_{1}+\frac{2\left(\alpha \beta_{3}\right)^{2}}{\bar{\zeta}}
$$

From (14d), there exists $\zeta>0$ such that $\Omega_{1}+\Omega_{2}>\zeta I$, and hence by choosing $\bar{\zeta}=\zeta$, we obtain

$$
\dot{V}(X(t), \eta(t)) \leq-\sigma V(X(t), \eta(t))+\chi|d(t)|^{2}
$$

with $\sigma:=\min \left\{\sigma_{1}, \sigma_{2}, \frac{\zeta}{4}\right\}$.

\section{Quantized Control}

We are interested in studying stabilization of the system (1), (2), (7) when the output $X(1, \cdot)$ is quantized using a set of finite alphabets, and cannot be transmitted to the control precisely. In this case, the role of disturbance $d$ is played by the quantization error. To define a quantizer, we first specify a set of finite alphabets $\mathscr{Q}:=\left\{q_{1}, q_{2}, \ldots, q_{N}\right\}$. A quantizer with sensitivity $\Delta_{q}>0$, and range $M_{q}>0$, is then a function $q: \mathbb{R}^{n} \rightarrow \mathscr{Q}$ having the property that 
Feedback Stabilization of Linear Hyperbolic Systems

$$
|q(x)-x| \leq \Delta_{q} \quad \text { if } \quad|x| \leq M_{q}
$$

and

$$
|q(x)| \geq M_{q}-\Delta_{q} \quad \text { if } \quad|x|>M_{q} .
$$

In other words, within the space $\mathbb{R}^{n}$, where the measurements of $X(1, \cdot)$ take values, we take a ball of radius $M_{q}$, and partition it into $N$ regions. Each of these regions is identified with a symbol $q_{i}$ from the set $\mathscr{Q}$. If $|X(1, t)| \leq M_{q}$, the controller receives a valid symbol and knows the variable $X(1, t)$, modulo the error due to sensitivity of the quantizer. When the measurements are out of the range of the quantizer, then the quantizer just sends an out of bounds flag and no upper bound on the error between $X(1, t)$ and its quantized value can be obtained in that case. For this paper, we limit ourselves to the case of static quantizers, that is, the parameters of the quantizer are assumed to be fixed which introduces a bounded measurement error determined by the sensitivity of the quantizer.

The ratio between the range and the sensitivity of the quantizer $M_{q} / \Delta_{q}$ determines the rate at which the information is communicated by the quantizer on average. The basic idea of the quantized control in finite-dimensional systems is to show that the state of the system converges to a certain ball around the origin if this rate is sufficiently large (to dominate the most unstable mode) [12]. In the same spirit, we derive a lower bound on the ratio $M_{q} / \Delta_{q}$ which is required to achieve practical stability in the presence of quantization errors.

With quantized measurements, the controller (7) takes the form

$$
\begin{aligned}
\dot{\eta}(t) & =-\alpha \eta(t)+\alpha q(X(1, t)) \\
u(t) & =K \eta(t) .
\end{aligned}
$$

By writing $q(X(1, t))=X(1, t)+q(X(1, t))-X(1, t)$, and letting $d_{q}(t):=q(X(1, t))-$ $X(1, t)$, we are indeed in the same setup as earlier with $y(t)=q(X(1, t))$. Here, $d_{q}$ is such that

$$
\left|d_{q}\right| \leq \sqrt{n}\left|d_{q}\right|_{\infty} \leq \sqrt{n} \Delta_{q}, \quad \text { if }|X(1, t)|_{\infty} \leq M_{q} .
$$

To state this result, we need the following lemma which relates $|X(1, t)|$ with the value of the Lyapunov function $V$ considered in the previous section and defined in (16).

Lemma 1. There exists a constant $C>0$ such that

$$
|X(1, t)|^{2} \leq C V(X(\cdot, t), \eta(t)), \quad \forall t \geq 0,
$$

for the Lyapunov function $V$ defined in (16).

The proof of Lemma 1 is omitted but we emphasize that the value of $C$ in (27) can be computed directly in terms of closed-loop system data, see [18] details.

Theorem 3. Consider system (1), (2) and (26), and assume that the conditions of Theorem 2 hold, and the initial condition $X^{0}$ and $\eta^{0}$ satisfy

$$
C V\left(X^{0}, \eta^{0}\right) \leq M_{q}^{2}
$$


where the constant $C$ is obtained from (27). With the constants $\sigma, \chi$ appearing in (23), if the quantizer is designed such that

$$
\frac{M_{q}^{2}}{\Delta_{q}^{2}}>\frac{C n \chi}{\sigma},
$$

then the following items hold:

- The output $X(1, t)$ remains within the range of the quantizer for all $t \geq 0$, that is,

$$
|X(1, t)| \leq M_{q}, \quad \forall t \geq 0 .
$$

- The state of the system remains ultimately bounded in $\mathscr{H}^{1}$-norm, that is, there exists $T$ such that for all $t \geq T$

$$
V(X(\cdot, t), \eta(t)) \leq \gamma_{q}\left(\Delta_{q}\right)
$$

where $\gamma_{q}(s)=\frac{n C \chi}{\sigma} s^{2}(1+\varepsilon)$, for some sufficiently small $\varepsilon>0$, is a class $\mathscr{K}$ function.

We provide below a sketch of the proof of Theorem 3, and suggest the reader to consult [18] for more details.

In the light of condition (29), fix $\varepsilon>0$ such that

$$
\frac{n \chi}{\sigma} \Delta_{q}^{2}(1+\varepsilon) \leq \frac{M_{q}^{2}}{C} .
$$

When the controller uses quantized measurements of $X(1, t)$, the derivative of the Lyapunov function in (23) satisfies, along the solutions to (1), (2), (26),

$$
\dot{V}(X(t), \eta(t)) \leq-\sigma V(X(t), \eta(t))+\chi|q(X(1, t))-X(1, t)|^{2} .
$$

Thus, for the chosen $\varepsilon>0$, if

$$
\frac{n \chi}{\sigma} \Delta_{q}^{2}(1+\varepsilon) \leq V(X(t), \eta(t)) \leq \frac{M_{q}^{2}}{C}
$$

then, using (27), $|X(1, t)| \leq M_{q}$ implying, with (24), that $|q(X(1, t))-X(1, t)| \leq$ $\sqrt{n} \Delta_{q}$, and hence

$$
\dot{V}(X(t), \eta(t)) \leq-\varepsilon n \chi \Delta_{q}^{2} .
$$

From the constraints imposed on the initial condition of the system, it readily follows from the above inequality that

$$
|X(1, t)|^{2} \leq C V(X(t), \eta(t)) \leq M_{q}^{2}, \quad \forall t \geq 0,
$$

and hence the quantization error is always upper bounded by $\Delta_{q}$. The uniform decrease in the value of $V$ also guarantees that 
Feedback Stabilization of Linear Hyperbolic Systems

$$
V(X(t), \eta(t)) \leq \frac{n \chi}{\sigma} \Delta_{q}^{2}(1+\varepsilon) .
$$

for sufficiently large $t$, and for initial condition satisfying $|X(1,0)| \leq M_{q}$. This concludes the sketch of proof of Theorem 3.

\section{Example}

To illustrate the effectiveness of the controller (7), we provide simulation results for the case of a $2 \times 2$ hyperbolic system. The system we simulate is of the form (1) with

$$
\Lambda:=\left[\begin{array}{ll}
1 & 0 \\
0 & 2
\end{array}\right], \quad S:=\left[\begin{array}{cc}
0.1 & 0 \\
0 & 0.3
\end{array}\right]
$$

and the boundary condition (2) is described by

$$
H=\left[\begin{array}{cc}
0.25 & -1 \\
0 & 1.25
\end{array}\right], \quad B=\left[\begin{array}{ll}
1 & 0 \\
0 & 1
\end{array}\right]
$$

We first check conditions for Theorem 2. Selecting the matrix $K=\left[\begin{array}{cc}0 & 0.5 \\ -0.25 & -0.5\end{array}\right]$, it could be checked that the conditions of Theorem 2 are satisfied with

$$
\mu=0.1, \quad \kappa=0.2, \quad D=\left[\begin{array}{ll}
1 & 0 \\
0 & 1
\end{array}\right], \quad \alpha=90, \quad \beta_{1}=1, \quad \beta_{2}=1, \quad \beta_{3}=75 .
$$

Thus the ISS estimate (8) holds for (11a) and (26) with the closed-loop boundary condition (11c). One can for example select the following initial condition, which satisfies the first-order compatibility condition for the existence of solutions in $\mathscr{H}^{1}\left((0,1) ; \mathbb{R}^{n}\right)$ :

$$
X_{1}(z, 0)=\cos (4 \pi z)-1, \quad X_{2}(z, 0)=\cos (2 \pi z)-1,
$$

with $z \in[0,1]$.

Now to illustrate Theorem 3, let us consider the quantizer centered at the origin, and given by

$$
q(x)=\lfloor\ell x+0.5\rfloor / \ell
$$

with the parameter $\ell=5$. The error due to quantization in this case is $\Delta_{q}=1 / \ell$, and for the sake of simplicity we take a sufficiently large range $M_{q}$ to bound the initial condition.

The time-evolution of the solutions for the first and second component of $X$, as well as the state of the dynamic controller $\eta$ are plotted in Figures 1 and 2 respectively. It could be seen that the solution to (11) converges to a neighborhood of the origin as the time increases. This simulation is thus in agreement with the result 
reported in Theorem 3. Figure $2 \mathrm{~b}$ also gives the time-evolution of the quantized measurements.

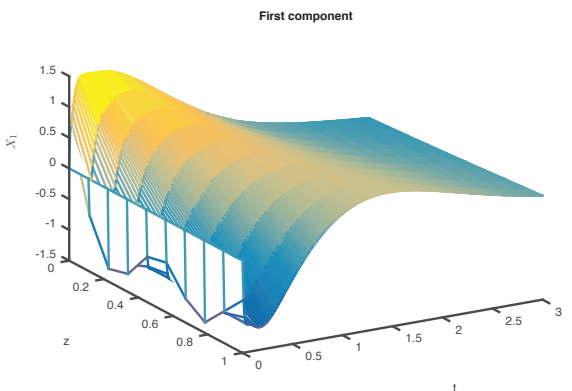

(a) Evolution of $X_{1}$

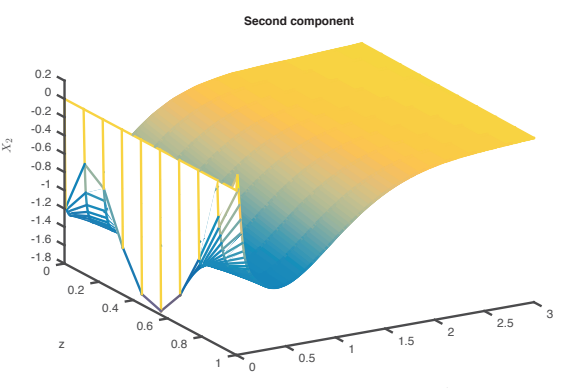

(b) Evolution of $X_{2}$

Fig. 1: Time and space evolution of state $X$.

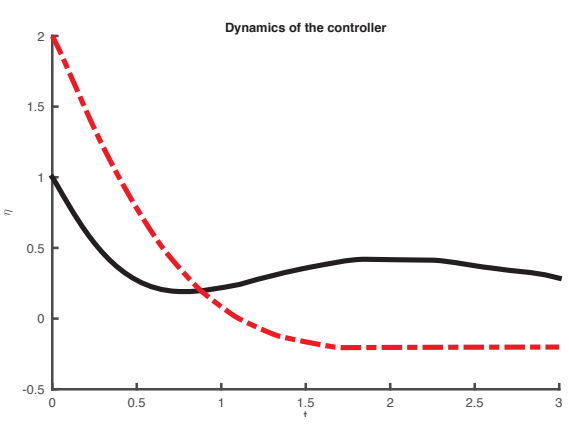

(a) Time evolution of $\eta$. Black: $\eta_{1}$, Red: $\eta_{2}$.

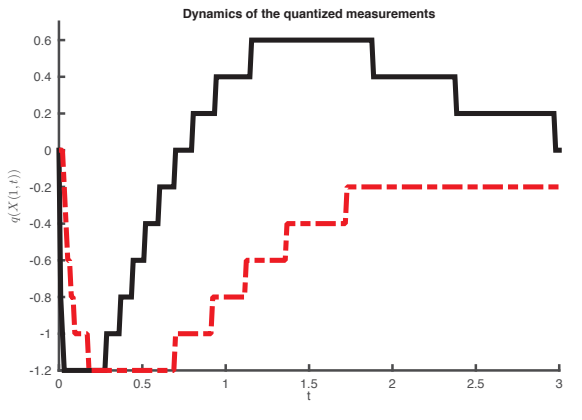

(b) Time evolution of $q(X(1, t))$. Black: $q\left(X_{1}(1, t)\right)$, Red: $q\left(X_{2}(1, t)\right)$

Fig. 2: Time evolution of controller state $\eta$ and quantized measurements $q(X(1, t))$.

\section{Conclusion}

In this chapter, the stabilization of partial differential equations described by linear balance laws when the measurements are subjected to disturbances has been addressed via the use of a dynamic controller. This chapter can then be complementary to our previous work on robust stabilization of linear hyperbolic systems, because the presence of source terms in the system description has been taken into 
account. As a first step, the case of static controllers has been tacked allowing to provide conditions on stability of the closed-loop system and robustness with respect to measurement errors. In a second step, the case of dynamic controllers is considered. Conditions in terms of matrix inequalities have been then proposed to bound the maximum norm of the state trajectory, leading to robust stability of the closed-loop system in the presence of measurement errors in the feedback. The case of the quantized control, where the quantization error plays the role of disturbance in the measurement, have been also considered.

This work opens the door for studying other problems. For example, it could be interesting to study the design of a more general dynamical controller ensuring the robustness properties of the closed loop.

\section{References}

1. G. Bastin and J.-M. Coron. Stability and Boundary Stabilization of 1-D Hyperbolic Systems. Subseries in Control: Progress in Nonlinear Differential Equations and Their Applications. Birkhauser, 2016.

2. G. Bastin, J.-M. Coron, and B. d'Andréa-Novel. Using hyperbolic systems of balance laws for modeling, control and stability analysis of physical networks. In Lecture notes for the PreCongress Workshop on Complex Embedded and Networked Control Systems, Seoul, Korea, 2008. 17th IFAC World Congress.

3. J.-M. Coron, G. Bastin, and B. d'Andréa Novel. Dissipative boundary conditions for onedimensional nonlinear hyperbolic systems. SIAM Journal on Control and Optimization, 47(3):1460-1498, 2008.

4. S. Dashkovskiy and A. Mironchenko. Input-to-state stability of infinite-dimensional control systems. Mathematics of Control, Signals, and Systems, 25(1):1-35, 2013.

5. N. Espitia, A. Girard, N. Marchand, and C. Prieur. Event-based control of linear hyperbolic systems of conservation laws. Automatica, 70:275-287, 2016.

6. I. Karafyllis and M. Krstic. ISS with respect to boundary disturbances for 1-D parabolic PDEs. IEEE Transactions on Automatic Control, 61(12):3712-3724, 2016.

7. I. Karafyllis and M. Krstic. ISS in different norms for 1-D parabolic PDEs with boundary disturbances. To appear, 2017.

8. D. Liberzon. Hybrid feedback stabilization of of systems with quantized signals. Automatica, 39(9):1543-1554, 2003.

9. F. Mazenc and C. Prieur. Strict Lyapunov functions for semilinear parabolic partial differential equations. Mathematical Control and Related Fields, 1(2):231-250, 2011.

10. A. Mironchenko and H. Ito. Construction of Lyapunov functions for interconnected parabolic systems: An iISS approach. SIAM Journal on Control and Optimization, 53(6):3364-3382, 2015.

11. A. Mironchenko and F. Wirth. A note on input-to-state stability of linear and bilinear infinitedimensional systems. In 54th Conference on Decision and Control, pages 495-500, 2015.

12. G. Nair, F. Fagnani, S. Zampieri, and R.J. Evans. Feedback control under data rate constraints: An overview. Proceedings of the IEEE, 95(1):108 - 137, 2007.

13. C. Prieur and F. Mazenc. ISS-Lyapunov functions for time-varying hyperbolic systems of balance laws. Mathematics of Control, Signals, and Systems, 24(1):111-134, 2012.

14. C. Prieur and A. Tanwani. Asymptotic stabilization of some finite and infinite dimensional systems by means of dynamic event-triggered output feedbacks. In Feedback Stabilization of Controlled Dynamical Systems, volume 473 of Lecture Notes in Control and Information Sciences, pages 201-230. Springer, 2017. 
15. E. D. Sontag. Smooth stabilization implies coprime factorization. IEEE Transactions on Automatic Control, 34(4):435 - 443, 1989.

16. A. Tanwani, C. Prieur, and M. Fiacchini. Observer-based feedback stabilization of linear systems with event-triggered sampling and dynamic quantization. Systems \& Control Letters, 94:46 - 56, 2016.

17. A. Tanwani, C. Prieur, and S. Tarbouriech. Input-to-state stabilization in $H^{1}$-norm for boundary controlled linear hyperbolic pdes with application to quantized control. In 2016 IEEE 55th Conference on Decision and Control (CDC), pages 3112-3117, Dec 2016.

18. A. Tanwani, C. Prieur, and S. Tarbouriech. Disturbance-to-state stabilization and quantized control for linear hyperbolic systems. IEEE Transactions on Automatic Control, 2017. To appear. Available at arXiv:1703.00302.

19. A. Tanwani, A.R. Teel, and C. Prieur. On using norm estimators for event-triggered control with dynamic output feedback. In Proc. 54th IEEE Conf. on Decision and Control, pages 5500-5505, 2015. 